\title{
Detection of Mycobacterium tuberculosis from sputum samples of outdoor patients in Comilla, Bangladesh
}

\author{
Baitul Islam, Md. Zahidul Islam, Tithi Rani Das and Tasnia Ahmed*
}

Department of Microbiology, Stamford University Bangladesh, 51, Siddeswari Road, Dhaka-1217, Bangladesh

\author{
Received 04 September 2019/Accepted 15 November 2019
}

\begin{abstract}
Tuberculosis (TB) caused by Mycobacterium tuberculosis has plagued humans since the beginning of history, but was on the decline after introduction of the Bacillus-Calmette-Guerin (BCG) vaccination programs. Tuberculosis appears to be an emerging problem worldwide due to environmental changes, mass migration and rise of drug-resistance phenomenon. This study targets to find out the distribution of TB patients in the sub-urban population around Comilla city in Bangladesh. A total of 455 patients with pulmonary symptoms were included in the study over a period of six months (from January 2016 to June 2016). Samples were smeared and stained with acid-fast technique and cultured in Lowenstein Jensen medium. A total of $2.4 \%$ of the patients were diagnosed with active pulmonary TB, $1.53 \%$ of whom are males and $0.87 \%$ are females. On the other hand, the Mantoux Tuberculin test showed $5.3 \%$ of these patients to be positive for $\mathrm{TB}, 3.3 \%$ of whom are males and $2 \%$ are females. $23 \%$ of the samples were sputum, $59 \%$ were pus, $14 \%$ were cerebrospinal fluid (CSF) and $4 \%$ were tracheal aspirates. On the whole, the suburban population around Comilla city in Bangladesh has a moderate incidence of pulmonary TB with military TB being rarely present. A thorough multi-year surveillance is needed to control the situation.
\end{abstract}

Keywords: Tuberculosis, Pulmonary Symptoms, Acid-fast Staining, Tracheal Aspirates.

\section{INTRODUCTION}

Tuberculosis (TB) is a contagious, wasting disease caused by any of several mycobacteria. The most common form of the disease is tuberculosis of the lungs (Pulmonary consumption or phthisis), but the intestines, bones and joints, the skin, and the genitourinary, lymphatic and nervous systems may also be affected. Tuberculosis is an infectious disease caused by the microorganism Mycobacterium tuberculosis. It mostly affects the lungs (Pulmonary Tuberculosis) with some involvement of other organs of the human body like the kidneys, the brain, and the bones in disseminative cases (1-4).

Mycobacterium tuberculosis is responsible for the infection named tuberculosis which is readily transmissible by breathing in infectious droplets expelled from the respiratory tract of another infected person (1-4). Though tuberculosis disease initiates with the damages in lungs, but in later cases when left untreated, it is possible for the bacterium to spread to any other organ or system which may cause death (5). Treatment with antibiotics is possible, but long term administration of medicine is necessary to achieve complete remission. TB primarily infects the lungs, but it may attack almost any tissue or organ of the body. The disease TB has an extended latency period and there is evidence that only $10 \%$ of infected people with normal immunity ever experience active TB (68 ). For people with immune deficiencies, active TB is much more common. TB is transmitted in close quarters when a person with active TB coughs the microbe into the air (6-8). TB is present with an extreme high prevalence in Asian countries, where
$60-80 \%$ children below the age of 15 years are infected (2). Bangladesh is sixth of the 22 countries enlisted by WHO as high burden countries (9-11). Estimates suggest that daily 880 new TB cases and 176 TB deaths occur in the Bangladesh $(6,9-10,12)$.

TB detection is critical for the proper management of the patients. The most traditional way to detect tuberculosis is a combination of chest $\mathrm{X}$-rays followed by acid-fast the staining of sputum for microscopic detection and finally growing the Mycobacterium tuberculosis in culture with the Mantoux test (13). Smear microscopy is the most simplest and rapid procedure currently available to detect acid fast bacilli in clinical specimen (12, 14-16). Isolation of organisms is the only currently available means for the diagnosis of the TB. The present study was conducted for the primary screening of $M$. tuberculosis by smear microscopy and Montoux test to initiate the treatment of the patients.

\section{MATERIALS AND METHODS}

Study settings. The study was conducted in the National Tuberculosis Reference laboratory of Combined Military Hospital (CMH), Comilla Cantonment. Being a tertiary hospital, $\mathrm{CMH}$ has the facilities for the diagnosis of TB in Comilla Cantonment and usually handles a significant number of TB patients.

Study population. A total of 455 patients of TB were enrolled in this study. Among the patients, 320 were male and 135 were female.

Ethical issue. Permission was taken from the administrative authority of Department of pathology \& Microbiology of Combined Military Hospital (CMH), Cumilla Cantonment. Permission from the patient legal guardian was taken prior collecting sample. The $\mathrm{CMH}$ ethics committee specifically approved this study.

Sample collection, smear preparation, staining and microscopic observation. Sputum, pus, CSF, tracheal aspirate samples were collected in cleaned, dry, wide-necked, leak-proof containers and smears were prepared from yellow purulent portion of the sputum using a sterile bamboo stick. The smear was spread evenly, air-dried for 15 minutes, and fixed by placing the 
slide over a hot plate at $85^{\circ} \mathrm{C}$ for 3 minutes $(9-10,12,17)$

Acid-Fast Procedure. Smears were prepared, heat-fixed, absorption pape (bibulous paper) above the smear was removed to fit the slide leaving one end for handling. The paper should not be allowed to protrude beyond the slide and the smears must be covered. The slide was placed on wire gauze on a ring stand. Slides were heated with a hand-held bunsen until steam could be seen rising from the surface. Alternately the burner was removed and reheated the slide to maintain steaming for 3-5 minutes. During the whole staining process, a drop or two of carbol fuschin was added time to time to prevent dryness and to keep the slide moist. It should be kept in mind to avoid adding excessive stain which might cool the slide (and drip on the bench). In the contrary, overheating also must not be allowed on the slide as letting it dry will distort the cells. Under heating will fail to stain acid-fas cells. The paper was removed with tweezers at the end of the staining and washed thoroughly. The slide was drained. The slide was washed with acidfast alcohol. The slide was rinsed, drained, and counterstained with methylene blue for 45 seconds. The slide was rinsed, blotted and examined. First each organism was observed on its separate smear. Then the mixed smear was examined. Acid-fast organisms appear as red and non-acid fast organisms as blue.

Screening by Mantoux tuberculin skin tests. The routine screening of high risk individuals suffering from tuberculosis, Mantoux tuberculin skin test is the most conventional method used. Among the 455 patients 150 were subjected to Mantoux tuberculin skin tests.

\section{RESULTS AND DISCUSSION}

Tuberculosis continues to be important contributor to overall disease burden and death in Bangladesh, with an estimated 46 positive cases in 1,00,000 suspected people and 73,000 deaths each year, Bangladesh ranks $5^{\text {th }}$ in the world in tuberculosis disease burden (18).

In collaboration with the National Tuberculosis Control Programmer (NTP), WHO, BRAC, Damien Foundation, Netherlands Tuberculosis Foundation (KNCV) USAID Bangladesh and other partners of NTP, icddr,b will be engaged in the survey including 50,000 individuals aged 15 years and older in 20 urban and rural clusters separately. In addition, icddr,b will also conduct a tuberculin survey among 20,000 children aged 5 to 14 years. The prevalence survey will improve estimates of TB cases and help in assessing the effect of TB control measures carried out by the NTP $(12,19)$.

455 samples collected from different areas from Comilla for sputum AFB, where $2.4 \%$ was positive. $0.87 \%$ was female and $1.53 \%$ was male (Table 1 ). For Mantoux tuberculin skin test 150 samples was collected. $5.3 \%$ positive samples were recovered among which $3.3 \%$ male and $2 \%$ was female (Table 2).

The WHO targets for TB control are to detect $70 \%$ of new smear-positive cases and to achieve a cure rate of $85 \%$. The NTP has achieved the cure rate target since 2003 and reached the $71 \%$ detection target in 2006. Estimates of the current TB situation in Bangladesh indicate an incidence of 227 new cases of all types and 102 new positive cases in smear test per 100,000 people in every year. Though, these calculations were estimated several years before, a specific updated estimation of the incidence of tuberculosis is urgently needed to determine the current progress in disease control and the degree of success in achieving WHO set targets (20).

Tuberculosis foundation of the Netherlands, KNCV is being provided with all necessary technical support for the survey by The World Health Organization.
Other NTP partners are also extending their full support and assistance in regard to conduct this survey successfully (20). While TB has long been a major problem in developing countries it was widely thought to have been eliminated in the developed world as a result of improved social conditions, mass screening, and effective use of antibiotics and the BCG vaccine (21). However, in the $1980 \mathrm{~s}$ it re-emerged in the West, for example in New York, where several thousand cases were reported. Rising poverty and homelessness, increased migration, overcrowding, drug abuse, and inadequate or reduced public health services are all contributing factors. In developed countries many research facilities have been closed, and screening programmers for disadvantaged groups, such as the homeless, are no longer readily available as was the case in previous decades (22). A major problem is the emergence of new, drug and antibiotic resistant strains of $\mathrm{TB}$ as a result of patients not completing courses of treatment because they feel better. Increasing numbers of people with AIDS (Acquired Immune Deficiency Syndrome) are developing symptoms of TB because of their lower resistance to disease.

Table 1. Number of TB positive patients according to sex by smear microscopy

\begin{tabular}{ccccc}
\hline Sex & $\begin{array}{c}\text { Total } \\
\text { Number }\end{array}$ & Positive & Negative & $\begin{array}{c}\text { Percentage } \\
(\boldsymbol{\%})\end{array}$ \\
\hline Male & 320 & 7 & 313 & 2.2 \\
Female & 135 & 4 & 131 & 3 \\
Total & 455 & 11 & 444 & 2.4 \\
\hline
\end{tabular}

Table 2. Number of TB positive patients according to sex by Mantoux tuberculin skin test

\begin{tabular}{ccccc}
\hline Sex & $\begin{array}{c}\text { Total } \\
\text { Number }\end{array}$ & Positive & Negative & $\begin{array}{c}\text { Percentage } \\
(\%)\end{array}$ \\
\hline Male & 92 & 5 & 87 & $5.4 \%$ \\
Female & 58 & 3 & 55 & $5.2 \%$ \\
Total & 150 & 8 & 142 & $5.3 \%$ \\
\hline
\end{tabular}

More people are infected with the disease today than at any other time in history. The World Health Organization (WHO) has estimated that 8 to 10 million people catch the issue every year, with 3 million dying from it. The death rate is significantly high for women with greater worldwide mortality rate than AIDS and malaria combined. TB 'black spots, include Eastern Europe, with 250,000 cases a year.The WHO predicts that if left unchecked TB will kill 35 million people in the world by 2025 . Long term goal in achieving reduced death rate of 50 percent by 2015 and elimination of the disease by 2025 has also been announced (23).

Multidrug resistant tuberculosis (MDR-TB) is one of the major public health concerns world worldwide particularly in developing countries, including Bangladesh. Thus far, there are no well-validated clinical guidelines for the prevention of MDR-TB. 
This study aims to evaluate the improvement in nurses practice using the newly developed Nursing practice Guidelines for the prevention of MDR-TB (NPG: MDR-TB) among hospitalized adults patients in Bangladesh. The guidelines were developed, disseminated and evaluated among 64 nurses by assessing nursing practice for the prevention of MDRTB during pre and post-implementation of the prevention of MDR-TB in case finding and post test mean scores of nursing practice for the prevention of MDR-TB in case finding and case holding were found in their level of wards, including Level 0 (non-TB), Level 1 (TB) and Level 2 (MDR-TB) $(P<0.001)$.This indicated that the guidelines might be applicable to reduce the development of MDR-TB in hospitals. However, this was a preliminary study with a limited time frame. Further valuation is, therefore, needed.

The studies are expected to result in information that wound inform WHO guidelines on simple, safe and effective regimens for the treatment of clinical severe infection and pneumonia in newborns and young infants in settings where referral is not possible. This study will also inform the inputs and process required to established outpatient treatment of infections in new-borns and young infants at health facilities near the one. A total of $2.4 \%$ of the patients were diagnosed with active pulmonary TB, $1.53 \%$ of whom were males and $0.87 \%$ are females. The Mantoux Tuberculin test and microscopic examination yielded confusing results. Modern techniques like immunofluorecence or PCR gave rapid confirmatory results but they could not be applied in rural or sub urban areas for lack of infrastructure and high cost. $5.3 \%$ of these patients to be positive for TB, 3.3\% of whom are males and $2 \%$ are females. $23 \%$ of the samples were sputum, $59 \%$ were pus, $14 \%$ were cerebrospinal fluid (CSF) and 4\% were tracheal aspirates. Disseminated or Military TB was mostly found in Bangladeshi suburban population.

\section{CONCLUSION}

We expect that the information from research and the resulting WHO guidelines will form the basis policy dialogue by a large number of stakeholders at the country level to implement outpatient treatment of neonatal infections and thereby reduce neonatal and infant mortality resulting from infections.

\section{ACKNOWLEDGEMENTS}

Authors are thankful to the Department of Microbiology, Stamford University Bangladesh for the technical supports provided during the study.

\section{REFERENCES}

1. Pawlowski A, Jansson M, Sköld M, Rottenberg ME and Källenius G. 2012. Tuberculosis and HIV co-infection. PLoS Pathog. 8:e1002464.

2. World Health Organization. 2010. Global tuberculosis control 2010. Geneva, Switzerland: World Health Organization Press.

3. Kumar V, Abbas AK, Fausto N and Mitchelll RN. 2007. Robbins Basic Pathology ( $8^{\text {th }}$ ed). Saunders Elsevier. 8:516-522.

4. McAdam RA, Quan S, Smith DA, Bardarov S, Betts JC, Cook FC et al. 2002. Characterization of a Mycobacterium tuberculosis H37Rv transposon library reveals insertions in $351 \mathrm{ORFs}$ and mutants with altered virulence. Microbiology. 148:2975-2986.

5. Simonovska L, Trajcevska M, Mitreski V and Simonovska I. 2015. The causes of death among patients with tuberculosis. Eur. Respir. J. 46:PA2713.

6. Rahman F, Munshi SK, Kamal SMM, Rahman ASMM, Rahman MM, Noor R. 2011. Comparison of different microscopic methods with conventional TB culture. Stam. J. Microbiol. 1:46-50.

7. Aurin TH, Munshi SK, Kamal SM, Rahman MM, Hossain MS, Marma T. 2014. Molecular Approaches for Detection of the Multi-Drug Resistant Tuberculosis (MDR-TB) in Bangladesh. PLoS ONE. 9:e99810.

8. Jeon K, Choi WI, An JS, Lim SY, Kim WJ, Park GM et al. 2012. Paradoxical response in HIV-negative patients with pleural tuberculosis: a retrospective multicentre study. Int. J. Tuberc. Lung. Dis. 16:846-51.

9. Noor R, Akhter S, Rahman F, Munshi SK, Kamal SMM, Feroz F. 2013. Frequency of extensively drug resistant tuberculosis (XDR-TB) among re-treatment cases in NIDCH, Dhaka, Bangladesh. J. Infect. Chemother. 19:243-248.

10. Noor R, Hossain A, Munshi SK, Rahman F and Kamal SMM. 2013. Slide drug susceptibility test for the detection of multi-drug resistant tuberculosis in Bangladesh. J. Infect. Chemother. 19:818-824.

11. Lutfor A. 2009. Extensively Drug Resistant Tuberculosis (XDR-TB). Bangladesh J. Med. Microbiol. 3:1-3.

12. Islam Z, Sanin KI and Ahmed T. 2017. Improving case detection of tuberculosis among children in Bangladesh: lessons learned through an implementation research. BMC Public Health 17:131.

13. Helb D, Jones M, Story E, Boehme C, Wallace E, Ho K et al. 2010. Rapid Detection of Mycobacterium tuberculosis and Rifampin Resistance by Use of On-Demand, Near-Patient Technology. J. Clin. Microbiol. 48:229-237.

14. Moore DA, Mendoza D, Gilman RH, Evans CA, Hollm Delgado MG, Guerra J et al. 2004. Tuberculosis Working Group in Peru.Microscopic observation drug susceptibility assay, a rapid, reliable diagnostic test for multidrug-resistant tuberculosis suitable for use in resource-poor settings. J. Clin. Microbiol. 42:4432-7.

15. Kommareddi S, Abramowsky C, Swinehart G and Hrabak L. 1984. Nontuberculous mycobacterial infections: comparison of the fluorescent auramine-O and ZiehlNeelsen techniques in tissue diagnosis. Hum. Pathol. 15:1085-1089.

16. Truffot-Pernot C, Véziris N and Sougakoff W. 2006. Modern diagnosis of tuberculosis. Presse. Med. 35:1739-1746.

17. Munshi SK, Rahman F, Kamal SMM and Noor R. 2012. Comparison among different diagnostic methods used for the detection of extrapulmonary tuberculosis in Bangladesh. Int. J. Mycobacteriol. 1:190-195.

18. Das AC. 2016. Epidemic situation of tuberculosis in Bangladesh: An overview. South East Asia J. Public Health 6:61-62

19. Hossain S, Zaman K, Quaiyum A, Banu S, Husain A, Islam A et al. 2014. Care seeking in tuberculosis: results from a countrywide cluster randomised survey in Bangladesh. BMJ. Open 4:e004766.

20. Zuniga ES, Early J and Parish T. 2015. The future for early-stage tuberculosis drug discovery. Future Microbiol. 10:217-229.

21. Bloom BR, Atun R, Cohen T, Dye C, Fraser H, Gomez GB et al. Tuberculosis. In: Holmes KK, Bertozzi S, Bloom BR, et al., editors. Major Infectious Diseases. 3rd edition. Washington (DC): The International Bank for Reconstruction and Development / The World Bank; 2017 Nov 3. Chapter 11.

22. Grant R, Gracy D, Goldsmith G, Shapiro A and Redlener IE. 2013. Twenty-Five Years of Child and Family Homelessness: Where Are We Now?. Am. J. Public Health 103:e1-e10.

23. Sandhu GK. 2011. Tuberculosis: current situation, challenges and overview of its control programs in India. J. Global Infect. Dis. 3:143150 